\title{
Correction to: PD1 inhibitor in combination with 5-azacytidine and low-dose DLI for the successful treatment of AML patients who relapsed after transplantation
}

Chong-Sheng Qian - Xiao Ma - Jin Wang • Ting-Jing Wang • Lian Bai • Hai-Xia Zhou • Ming-Zhu Xu • Ai-Ning Sun • De-Pei Wu $\cdot$ Song-Bai Liu $\cdot$ Sheng-Li Xue 10

Published online: 19 April 2021

(c) The Author(s), under exclusive licence to Springer Nature Limited 2021

Correction to: Bone Marrow Transplantation

https://doi.org/10.1038/s41409-020-01130-8
Unfortunately, in the original published version, the representation of Fig. 1 was incorrect. 
The correct figure is given below.

Fig. 1 Flow chart and process of treatment. a Flow chart of the use of a PD1 inhibitor in combination with 5-azacytidine and DLI. b Treatment process of case 1. $\mathbf{c}$ Treatment process of case 2. d PD1 expression on $\mathrm{T}$ cells during treatment.

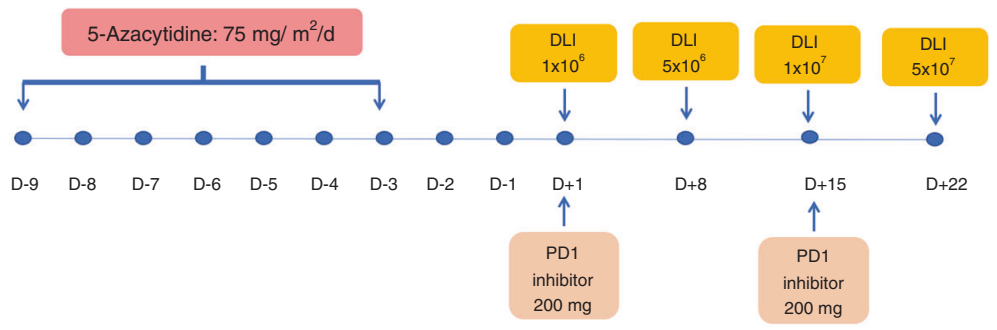

b
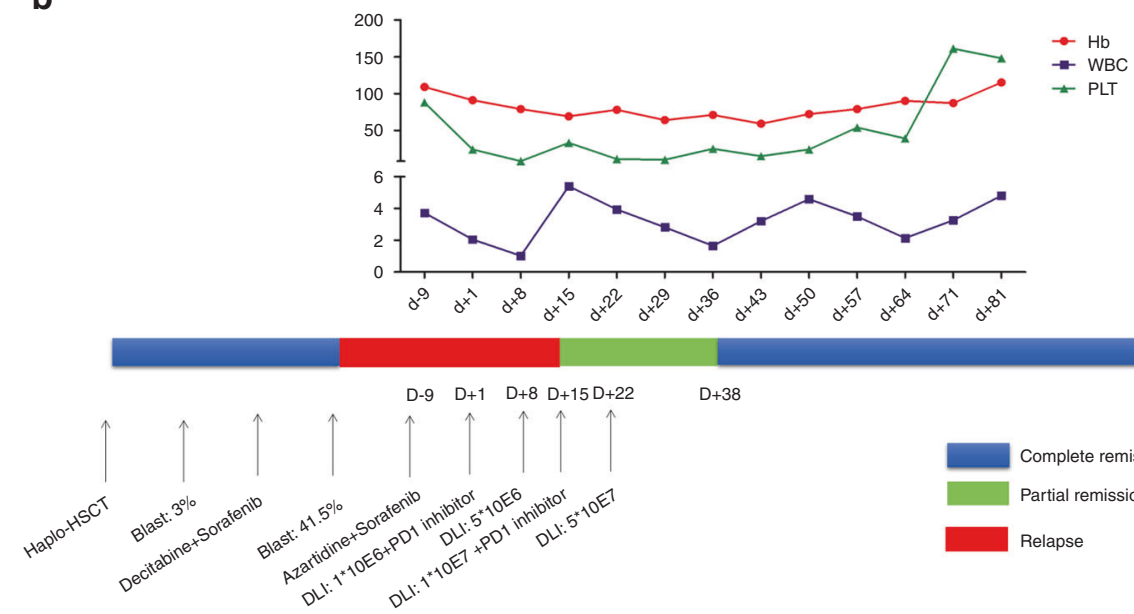

D+257

Complete remission

Partial remission

Relapse

C

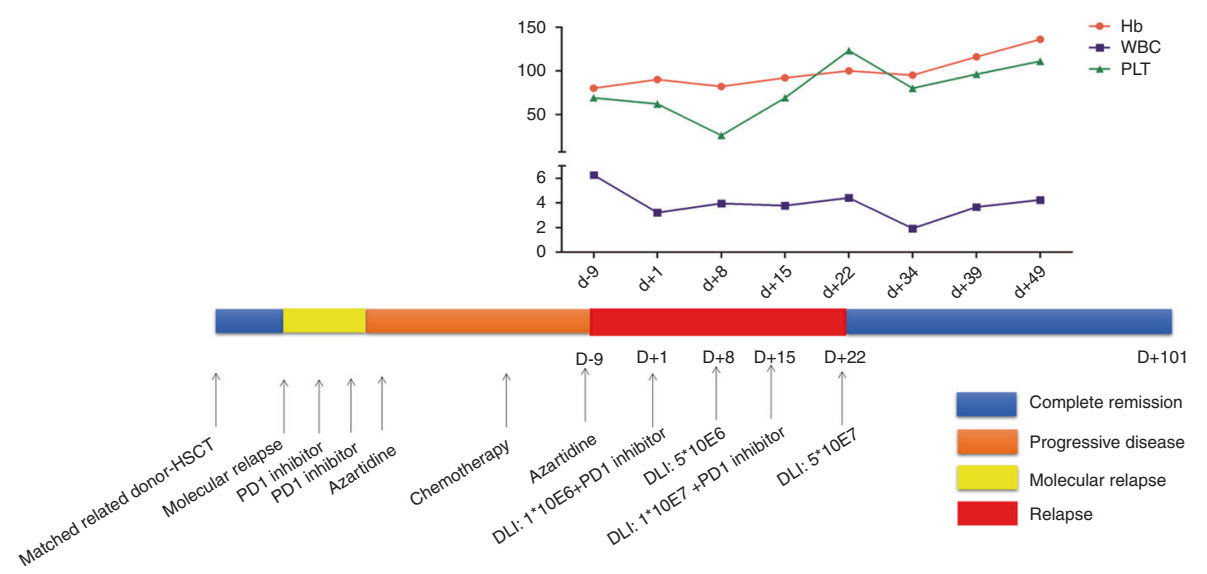

d

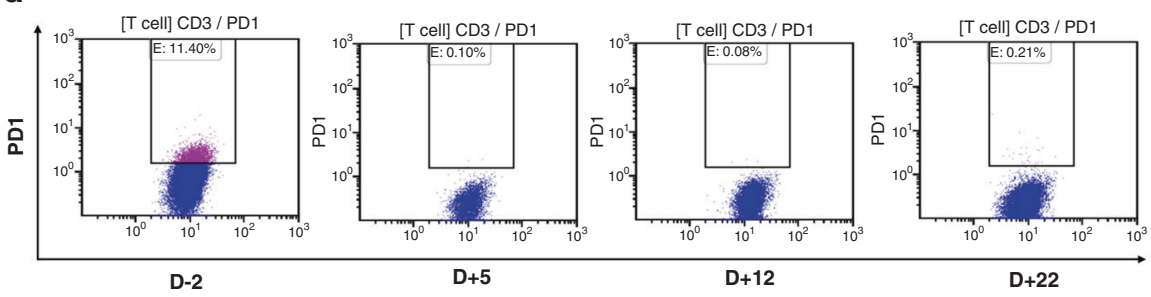

The original article has been corrected. 